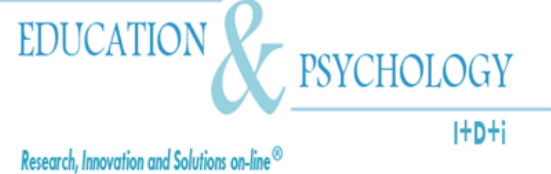

\title{
Equipos de Orientación Educativa en la Comunidad andaluza: análisis de la situación actual en la provincia de Huelva
}

\author{
María Cinta Aguaded Gómez ${ }^{1}$, Antonio Rus Arboledas ${ }^{2}$, José \\ Antonio Ávila Fernández ${ }^{3}$
}

\footnotetext{
${ }^{1}$ Departamento de Educación, Universidad de Huelva

${ }^{2}$ Departamento de psicología educativa y de la educación, Universidad de Granada

${ }^{3}$ Departamento de Educación, Universidad de Huelva
}

\section{España}

Correspondencia: María Cinta Aguaded Gómez. Universidad de Huelva, avda 3 de Marzo, s/n. 21007 Huelva.España. E-mail: mariacintaaguaded@dedu.uhu.es 


\section{Resumen}

Introducción. El debate territorial sobre la orientación educativa ha avanzado por diferentes caminos. En la comunidad andaluza se opta por conservar los equipos de orientación educativa como servicios externos de apoyo a los centros escolares de educación infantil y primaria.

Objetivo. El propósito de esta investigación es analizar variables diversas para acercarnos a la realidad que envuelve a estos servicios de apoyo a la orientación en los centros y realizar propuestas de mejora en torno a las aportaciones que nos vislumbra el modelo teórico que se utiliza en base a la titulación o las competencias que estos profesionales consideran importantes para llevar a cabo la intervención haciendo diferenciación por el género.

Método. Con la participación de todos los profesionales que trabajan en los EOEs de la provincia de Huelva, en sus distintos perfiles se obtuvo información a través de un cuestionario y grupo de discusión referida a diversas variables y el cruce de las mismas.

Resultados. Los resultados arrojan que en la función orientadora en sus distintos perfiles existe una marcada presencia de la mujer; el modelo ecléctico es el más usual en la intervención psicopedagógica; existe una diferencia de percepción según el género sobre las competencias necesarias para el desempeño para el puesto, así como una diferencia de opinión según la experiencia en el puesto en relación a las funciones más relevantes para ejercer la profesión

Discusión. Es de destacar como conclusión la feminación de la profesión orientadora. El predominio del modelo de intervención sobre el clínico, modelo más frecuente en investigaciones anteriores, esto supone un giro importante en la forma y procedimiento a la hora de actuar, las funciones que consideran prioritarias para desempeñar el puesto y las competencias más relevantes para ser un buen orientador, basadas más en características personales que en poseer una formación teórica.

Palabras Clave: Orientación, equipos de orientación educativa, equipo de apoyo externo, tutoría, asesoramiento y apoyo educativo

Recibido: 01/10/09 Aceptación Inicial: 14/10/09 Aceptación Definitiva: 27/01/10 


\title{
Educational Guidance Teams in Andalusia: an analysis of the current situation in Huelva
}

\begin{abstract}
Introduction. The territorial debate on educational guidance has moved along different paths. In the Andalusian community is chosen to maintain educational counseling teams as external services to support schools in early and primary education.

Objective. The purpose of this research is to analyze different variables for getting closer to the reality that surrounds these support services for guidance in schools and make proposals for improvement regarding the contributions that we glimpse to the theoretical model used on the basis of qualifications or skills that these professionals consider important for carrying out the intervention by gender differentiation.
\end{abstract}

Method. With the participation of all professionals working in the EOES of the province of Huelva, in their different profiles information was obtained through a questionnaire and group discussion concerning various variables and the crossing of them.

Results. The results show that in the guide role in their different profiles there is a marked presence of women, the eclectic model is the most common in psycopedagogy intervention; there is a difference in perception by gender on the competencies required to perform the job as well as a difference of opinion based on experience in the position, in relation to the functions most relevant to practice the profession.

Discussion. It should be noted, in conclusion, a feminization of the guiding profession. On the other hand, the eclectic model predominates over the clinical research, which was a most usual model in previous investigations, this represents a significant shift in the manner and procedure in time to act, the functions identified as priorities for the job and the skills most relevant to being a good counselor, based more on personal characteristics that have received training in theory.

Keywords: Counseling, education-oriented teams, external support team, tutoring, counseling and educational support.

Received: 10/01/09 Initial Acceptance: 10/14/09 Definitive Acceptance: 01/21/10 


\section{Introducción}

Para resaltar los aspectos novedosos que diversas leyes han aportado para la configuración del proceso de orientación y los servicios de apoyo a los centros se hará una aproximación histórica de la orientación desde la ley general de educación de 1970 hasta la actualidad, resaltando la ley orgánica general del sistema educativo y la ley orgánica de educación, por ser claves para el proceso de constitución actual.

A lo largo de este documento se va a hacer un recorrido sobre el contenido de la legislación en lo referente a la orientación; se comenta la ley general de educación, la institucionalización de los servicios de orientación en las comunidades autónomas, el desarrollo de la tutoría y los departamentos de orientación por la ley orgánica general del sistema educativo, concluyendo la revisión legal con unas reseñas sobre la regulación legislativa más cercana, la ley orgánica de educación y, en nuestro contexto autonómico, la ley de educación de Andalucía.

\section{Aproximación histórica}

\section{La ley general de educación}

En el sistema educativo se observa una evolución en la que poco a poco se van consolidando los servicios y actividades de orientación. Aunque anteriormente hayan existido antecedentes de la actividad orientadora, es la ley del 70 la que aboga plenamente por la orientación y las funciones tutoriales, aunque la falta de recursos económicos y formativos hizo que no se pusiera en práctica.

La promulgación, por tanto, de la ley general de educación (1970), fue de gran importancia para el sistema educativo español; en ella aparece la orientación, a lo largo de su articulado, como un referente de primer orden y con una clara dimensión educativa. Se reconoce por primera vez el derecho de los alumnos a la orientación educativa como un servicio continuado a lo largo de todo el proceso educativo. Se entiende la orientación como una actividad esencial de éste, dirigida al desarrollo integral del alumno individual y socialmente considerado, y consistente en ayudarle en el conocimiento, aceptación y dirección 
de sí mismo de acuerdo con sus circunstancias personales para facilitarle la toma de decisiones de un modo consciente y responsable, el desarrollo equilibrado de su personalidad, así como su incorporación comunitaria y al mundo del trabajo (Aguaded, 2009). "El planteamiento es asumir desde la participación de los padres, familia, docentes y comunidad en general la orientación del escolar como un todo" (Molina y De Luca, 2009: 1456).

Es pues, a partir de 1970, cuando se concibe propiamente un planteamiento general e institucional, con su correspondiente cobertura legal, de la orientación educativa en el sistema educativo español (Hernández Pina, 1988). Así, entre 1970 y 1985 se realizaron algunos esfuerzos para hacer llegar los servicios de orientación educativa y psicopedagógica a todo el país. Se adoptaron decretos, ordenes y resoluciones ministeriales, etc., en apoyo del establecimiento, parcial aunque insuficiente, de servicios de orientación para los ciclos de enseñanza primaria y secundaria. Sin embargo, si consideramos a toda la población estudiantil, estas disposiciones no proporcionaron solución alguna (Rodríguez, 1988).

El mayor hito de este periodo es la creación de los servicios de orientación educativa y vocacional. Fueron ellos, los que en definitiva, marcaron el inicio de los equipos externos de sector que posteriormente se difundieron desde distintas instancias. En enseñanzas medias cabe destacar la creación de los institutos de orientación educativa y profesional, a través del Real Decreto 2689, de 21 de noviembre de 1980, BOE, de 26/XII/80. Estaban destinados a funcionar solamente en los institutos y se encargaron del asesoramiento y orientación de corte institucional. Se les encomienda la colaboración con el personal docente de los centros de bachillerato y formación profesional para desarrollar tareas de orientación, se les insta a que se coordinen con los servicios de orientación educativa vocacional que operan en la educación general básica.

Esta iniciativa no tuvo la importancia que se pretendía, ya que fue un recurso poco extendido, y la amplitud de sus actuaciones y de sus funciones junto a la escasa dotación del personal y presupuestaria, las convirtió en un servicio muy limitado, especialmente en el terreno de apoyo y asesoramiento escolar.

La ley de integración social del minusválido (Ley 13 del 1982 del 7 de abril)

La ley de integración social del minusválido prevé en su título IV, artículo 10.I, 
referido al diagnóstico y valoración de las minusvalías, la creación de "equipos multidisciplinares" que, actuando en un ámbito sectorial, aseguren una atención interdisciplinaria a cada persona que lo precise, para garantizar su integración en su entorno socio comunitario.

El Real Decreto 334/1985, de 6 de marzo, de ordenación de la educación especial hace un nuevo planteamiento contemplando más ampliamente la problemática educativa de los disminuidos, disponiendo la dotación a los centros ordinarios de servicios que garanticen la integración. Entre ellos, la ampliación y desarrollo de los equipos.

En base a esta disposición legal se crearán equipos multidisciplinares centrados en la valoración y calificación de minusvalía "a efectos del reconocimiento de la prestación económica prevista en la ley en el instituto nacional de servicios sociales (INSERSO)" (López García, 1990: 521).

La ley 1/1990 de 3 de octubre, ley de ordenación general del sistema educativo, establece un modelo organizativo y funcional estructurado en tres niveles de intervención:

De aula, a través de la acción tutorial.

De centro, a través de los departamentos de orientación.

Del sistema escolar, a través de los profesionales de equipos psicopedagógicos que prestan el apoyo técnico a la institución escolar en su conjunto. La ley de ordenación general del sistema educativo, en definitiva, fomenta el desarrollo de la tutoría y del departamento de orientación del centro, así como el funcionamiento de equipos interdisciplinares que apoyan y dan soporte técnico a los anteriores.

La transferencia a las distintas comunidades autónomas de las competencias en el campo educativo, y por ende en el campo de la orientación, hace que cada territorio legisle independiente, apareciendo en cada uno de ellos un mosaico de modelos muy diversos (Rus, 1995).Con el diseño de un estado autonómico y la firma por parte del estado español de la carta social europea (1986), la orientación educativa empieza a surgir como un modelo institucionalizado de servicio de apoyo diferenciado en consonancia con lo que predomina en la unión europea (Rus, 1996). 


\section{Las transferencias educativas a las comunidades autónomas}

La institucionalización de los servicios de orientación en las distintas comunidades se inició en la década de los ochenta y se tomaron como precedentes: la ley general de educación del 70 con la creación de los servicios de orientación educativa y profesional en el 77, los IOEP (los institutos de orientación educativa y profesional) en 1980 y la LISMI (Ley de integración social del minusválido) en el marco de la educación especial, con la creación en 1982, de los equipos multidisciplinares.

La Junta de Andalucía al ver transferidas sus competencias en materia educativa va creando sus propios equipos con peculiaridades definidas, entre ellos, podemos destacar los siguientes:

Por el Decreto 238/1983 de 23 de noviembre de 1983 (Boletin Oficial del Estado, BOJA, 3/XII/83) se crean en esta comunidad los equipos de promoción educativa.

Los servicios de apoyo escolar se gestaron al amparo del Real Decreto 1.174/1983 de 27 de abril por el que se pone en marcha el programa de educación compensatoria y para desarrollar el Decreto 168/84 de 12 de junio (BOJA, 22/VI/84).

Durante el curso 1986/87 aparecen los equipos de atención temprana y apoyo a la integración, para intervenir sobre los alumnos diagnosticados y con discapacidad o deficiencia.

\section{La fusión. Equipos de orientación educativa}

Debido a los acontecimientos que se vivieron en etapas anteriores, en las que se reivindicaba una red única de orientación en la que se unificaran todos los servicios; las administraciones empezaron a legislar para presentar un modelo de orientación de Andalucía que respondiera a estas demandas.

Los servicios que habían aparecido a lo largo de la década de los ochenta, Equipos de Promoción y Orientación Educativa (EPOE), Servicios de Apoyo Escolar (SAE) y Equipos de Atención Temprana y Apoyo a la Integración (EATAI), para dar respuesta a aspectos concretos -integración, tutoría, orientación, compensatoria- incidían con frecuencia sobre la misma población escolar, produciéndose interferencias y solapamientos que amenazaban el 
beneficio que el alumnado podía obtener de su Intervención. El Decreto 213 de 1995 unifica estos servicios denominándolos equipos de orientación educativa quedando configurados definitivamente en un único ente.

\section{La Orientación hoy: LOE y LEA}

Las recientes publicaciones de las leyes que diseñan nuestro sistema educativo, tanto a nivel estatal como autonómico, así como su desarrollo legislativo correspondiente, hacen necesario plantear los contenidos de las mismas en relación con el tema a debate, los equipos de orientación y la orientación y la acción tutorial.

\section{Ley orgánica de educación 2/2006, de 3 de mayo}

La ley orgánica de educación, ya en su artículo 1, al enumerar los principios de la educación recoge en el apartado f) La orientación educativa y profesional de los estudiantes, como medio necesario para el logro de una formación personalizada, que propicie una educación integral en conocimientos, destrezas y valores. De la misma manera, en la redacción de los fines hacia los que se orientará el sistema educativo español, contenidos en el artículo 2, se establece en su apartado 2, que los poderes públicos prestarán una atención prioritaria al conjunto de factores que favorecen la calidad de la enseñanza y, en especial, la cualificación y formación del profesorado, su trabajo en equipo, la dotación de recursos educativos, la investigación, la experimentación y la renovación educativa, el fomento de la lectura y el uso de bibliotecas, la autonomía pedagógica, organizativa y de gestión, la función directiva, la orientación educativa y profesional, la inspección educativa y la evaluación, quedando incluida la orientación como rasgo de calidad de la enseñanza, como ya se había establecido en leyes anteriores.

Indica la ley, en el artículo 91 que la tutoría de los alumnos, la dirección y la orientación de su aprendizaje y el apoyo en su proceso educativo, en colaboración con las familias es una de las funciones del profesorado, no dejando a éste solo ante la tarea orientadora cuando se establece que la orientación educativa, académica y profesional de los alumnos, se realizará en colaboración, en su caso, con los servicios o departamentos especializados. Entre las competencias del claustro de profesores enumeradas en el artículo 129 se cita la de fijar los criterios referentes a la orientación, tutoría, evaluación y recuperación de los alumnos. 
Como se puede deducir, no se realiza un desarrollo exhaustivo de los aspectos orientadores y las estructuras organizativas que van a abordar la orientación, pues, como se establece en el artículo 157, al disponer sobre los recursos para la mejora de los aprendizajes y apoyo al profesorado, se indica que corresponde a las administraciones educativas proveer los recursos necesarios para garantizar, en el proceso de aplicación de la presente ley: h) La existencia de servicios o profesionales especializados en la orientación educativa, psicopedagógica y profesional, dejando una puerta abierta a la contextualización de los servicios y establecimiento de las estructuras necesarias que se estimen en las distintas comunidades autónomas.

\section{Ley de Educación de Andalucía 17/2007 de 10 de diciembre,}

En el apartado II de la exposición de motivos de la ley de educación de Andalucía se hace indicación a que la transformación del sistema educativo ha sido posible gracias a la ampliación de recursos, tanto humanos como materiales, dedicados a la formación permanente del profesorado y a la orientación educativa, refiriéndose a la red de formación y de orientación, consolidada, de que dispone la comunidad autónoma. Asimismo en el apartado IV se alude al capítulo III, donde se regulan los órganos colegiados de los centros, recogiendo entre ellos los órganos de coordinación docente y de orientación.

En el artículo 5, donde se plantean los objetivos de la ley, se cita: "potenciar la orientación educativa como medio para el desarrollo personal y como garantía de una respuesta educativa ajustada a las necesidades del alumnado", y en el artículo 7 sobre los derechos del alumnado, indica entre otros, la orientación educativa y profesional.

El capítulo III trata los órganos colegiados de gobierno y de coordinación docente, mencionando a los órganos de coordinación docente y de orientación en los centros públicos, $y$ regulando su funcionamiento en el artículo 13 en el que se indica que "son órganos de coordinación docente y de orientación... los equipos de ciclo y los equipos de orientación en los centros que imparten educación infantil y primaria...”, estableciéndose en el artículo 138 sobre "los equipos de ciclo y de orientación, que los centros públicos de educación infantil y primaria tendrán un equipo de orientación que estará integrado, al menos, por un profesional del equipo de orientación educativa..." En este momento de la ley se menciona por primera 
vez la organización de la orientación educativa en la educación infantil y primaria aludiendo a la presencia de los equipos multiprofesionales externos. Se concluye con la enumeración de competencias del equipo de orientación “que asesorará sobre la elaboración del plan de orientación y acción tutorial, colaborará con los equipos de ciclo en el desarrollo del mismo, especialmente en la prevención y detección temprana de las necesidades específicas de apoyo educativo, y asesorará en la elaboración de las adaptaciones curriculares para el alumnado que las precise".

Se realiza una mención en la LEA a la orientación en el artículo 126 al determinar la estructura del plan de centro, que se trata más adelante. El artículo 144, que trata de los servicios de apoyo a la educación, nombra a los equipos de orientación educativa, que los define como "unidades básicas de orientación psicopedagógica que, mediante el desempeño de funciones especializadas en las áreas de orientación educativa, atención al alumnado con necesidades específicas de apoyo educativo y apoyo a la función tutorial del profesorado, actúan en el conjunto de los centros que se determinen".

\section{El plan de centro y los elementos de orientación}

Regresando al artículo 126 de la ley de educación de Andalucía, que se ha mencionado anteriormente, en el que se trata del plan de centro, se observa en la siguiente figura que forma parte, de este primero, el plan de orientación y acción tutorial. Se expone, a continuación, en la figura 1.

Se entiende por "plan de orientación y acción tutorial... el instrumento pedagógicodidáctico que articula, a medio y largo plazo, el conjunto de actuaciones de los equipos docentes y del centro educativo en su conjunto, relacionados con la orientación y la acción tutorial." (Barrera et al, 2008: 14).

Con la inclusión en el plan de centro del plan de orientación y acción tutorial, se integran definitivamente los órganos de orientación y, en educación infantil y primaria, a los equipos de orientación educativa en la organización de los centros escolares (Ávila, 2009), dándose cuenta de uno de los aspectos ya incluidos en la orden de 16 de noviembre de 2007, que establece la orientación y acción tutorial, como: "la tarea colaborativa que, coordinada por la persona titular de la tutoría y asesorada por el orientador u orientadora de referencia, compete al conjunto del equipo docente del alumnado de un grupo". 
Tras esta aproximación histórica, factible de ampliación, se dará paso al proceso de investigación seguido, centrado en el estudio descriptivo de los equipos de orientación educativa, fórmula empleada en la comunidad autónoma de Andalucía para dar respuesta a las necesidades de orientación a docentes, alumnado y familias desde la organización externa como equipos multidisciplinares.

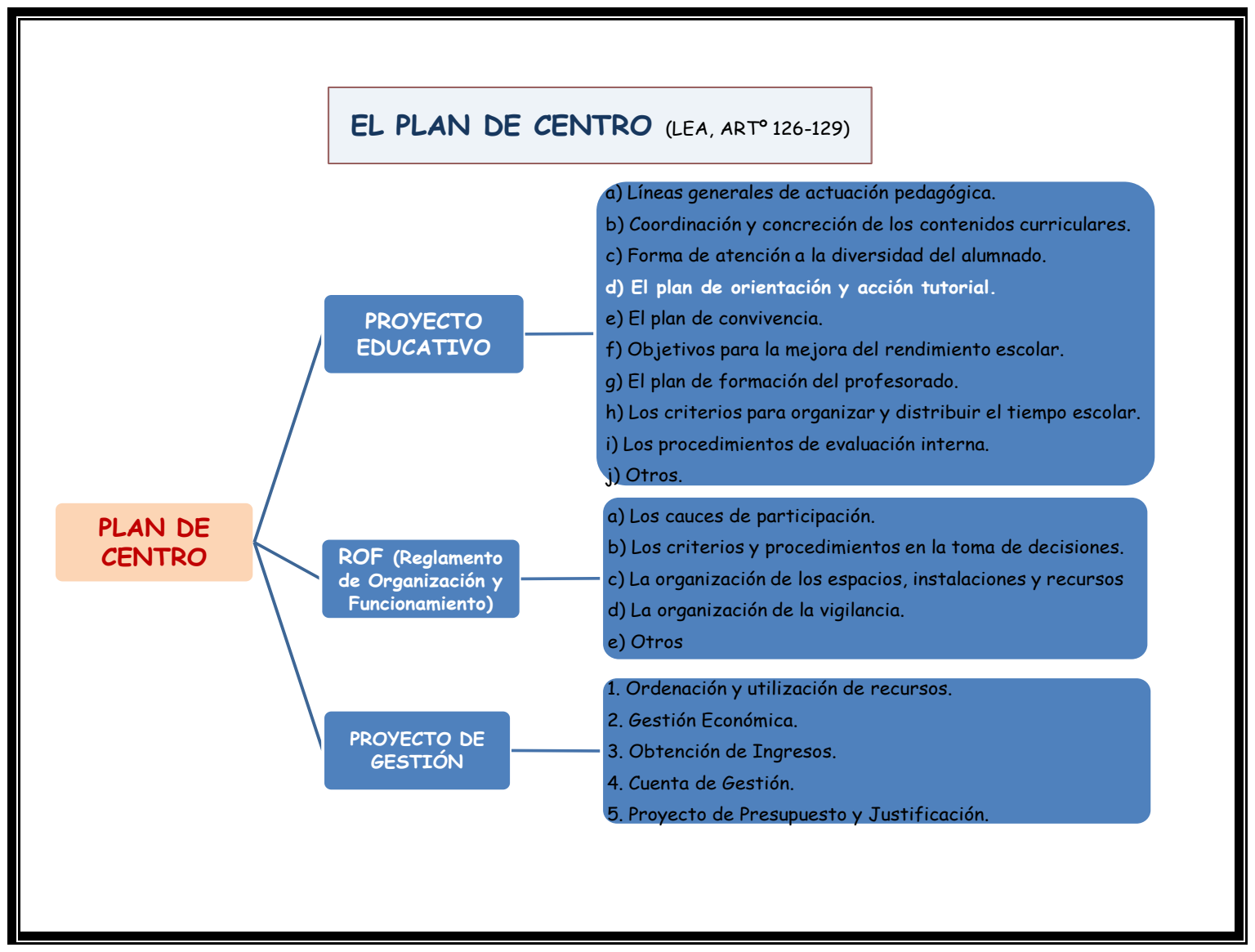

Figura 1. El plan de centro según la LEA.

El objetivo de esta investigación es exponer las conclusiones obtenidas mediante el cruce de variables estudiadas, como: género y titulación, género y competencias para el puesto, modelos teóricos y su relación con la titulación y años de experiencia y funciones de los equipos. Esto nos ayudará a entender la proyección de estos equipos, tanto en sus aspectos estructurales, como en la incidencia en los centros docentes. 


\section{Método}

\section{Participantes}

Una vez que hemos concretado nuestra investigación es necesario precisar "el campo"; esto es: "el contexto físico y social en el que tienen lugar los fenómenos objeto de la investigación” (Rodríguez et al., 1996, p. 45).

Nuestro estudio sobre la orientación educativa en la provincia de Huelva se centra en los servicios de apoyo externo a los centros. Quiere contribuir a enriquecer la definición de los equipos, como viejo pilar determinante de la orientación educativa en el sistema escolar. La población es la constituida por los profesionales de los equipos que actualmente trabajan en la provincia de Huelva durante el curso 2007/2008, un total de 94 sujetos.

En nuestro caso, la muestra invitada está compuesta por todos los miembros de equipos psicopedagógicos que desempeñan su labor en Huelva y provincia y la aceptante ha sido el $100 \%$ y la productora de datos que ha sido de 99\%. Los integrantes de la investigación fueron todos los profesionales en sus distintos pérfiles que componen los equipos de orientación educativa en la provincia de Huelva durante los curso 2007-2008.

\section{Instrumentos}

Este trabajo se sitúa dentro de los parámetros de la investigación cuantitativa, por lo tanto, por la naturaleza de nuestro estudio nos decantamos por el paradigma cuantitativo. Utilizaremos muchos de los instrumentos que le son propios, como los cuestionarios.

En esta línea, esta investigación “combina la recogida de información, a través de cuestionario para su procesamiento posterior, con el estudio de la realidad en el contexto natural, tal y como sucede, intentando sacar sentido de, o interpretar, los fenómenos de acuerdo con los significados que tienen para las personas implicadas" (Rodríguez et al., 1996: 32).

Entendemos que "la investigación por encuesta es una rama de los investigación social científica" (Kerlinger, 1979, p. 123). Además, el cuestionario resulta útil para conocer 
opiniones y actitudes (Pérez, 1988), con lo que podría ofrecer referencias para mejorar la intervención por parte de los equipos.

Con el objeto de encontrar el instrumento adecuado, analizamos varios cuestionarios referidos a equipos psicopedagógicos de otros autores que habían realizado investigaciones con características similares: Rus (1995), Hernández (2002), Fernández (2003). Ninguno cubría plenamente los objetivos de nuestra investigación, por ende decidimos en base a todo lo anterior construir nuestro propio cuestionario, que fue redactado como boceto de forma provisional. En la tabla siguiente, se recoge la estructura general. Cada dimensión se subdivide en sub-dimensiones que configuran las variables (ítems) y contienen la información precisa para completar y satisfacer toda la información acerca al objetivo planteado.

Tabla 1. Estructura del cuestionario

\begin{tabular}{|c|c|}
\hline DIMENSIONES & SUBDIMENSIONES O VARIABLES \\
\hline Datos de identificación & Ítems de 1 a 11 \\
\hline $\begin{array}{c}\text { Equipos de Orientación } \\
\text { Educativa }\end{array}$ & $\begin{array}{l}\text { 2.1. Funciones. Ítem } 12 \text {. } \\
\text { 2.2. Contenidos. Ítem } 13 \text {. } \\
\text { 2.3. Carácter multidisciplinar. Ítems 14-15-16-17. } \\
\text { 2.4. Competencias para el puesto. Ítems } 18 \text {. } \\
\text { 2.5. Intervención en los centros } \\
\text { 2.5.1. Plan Anual de zona. Ítems } 19 \text { y } 20 \\
\text { 2.5.2. Demandas. Ítems } 21 \text { al } 30 \\
\text { 2.6. Itinerancia. Ítems } 31 \text { y } 32\end{array}$ \\
\hline Equipo de zona & $\begin{array}{l}\text { 3.1. Recursos. Ítems de } 33 \text { a } 37 \\
\text { 3.2. Reparto de funciones. Ítems de } 38 \text { a } 41 \\
\text { 3.3. Horarios. Ítems de } 42 \text { a } 45\end{array}$ \\
\hline Coordinación & $\begin{array}{l}\text { 4.1. Zona. Ítems de } 46 \text { a } 48 \\
\text { 4.2. interzonal. Ítems de } 49 \text { a } 52 \\
\text { 4.3. Interprovincial-nacional e internacional. Ítems de } 53 \text { - } 54 \text {. } \\
\text { 4.4. Otras instituciones. Ítem } 55 \\
\text { 4.5. Inspección. Ítems del } 56 \text { al } 59 \\
\text { 4.6. Centro de Profesores. Ítems } 60 \text { al } 62 \\
\text { 4.7. Departamento de Orientación. Ítems } 63 \text { al } 65\end{array}$ \\
\hline El Equipo Técnico & Ítems del 66 al 70 \\
\hline Formación & Ítems del 71 al 75 \\
\hline $\begin{array}{l}\text { Valoración del puesto de } \\
\text { trabajo }\end{array}$ & Ítems del 76 al 82 \\
\hline
\end{tabular}


Como se puede apreciar en la tabla adjunta, el cuestionario consta de 7 dimensiones subdividido en varios sub-dimensiones. Cada una de ellas responde a un objetivo planteado en nuestra investigación.

\section{Procedimiento}

La naturaleza de esta investigación es eminentemente de tipo descriptiva y exploratoria, aunque también contiene elementos valorativos y prospectivos. Se trata de un primer acercamiento a la realidad del asesoramiento, desde nuestro contexto particular, que pretende ofrecer una panorámica general de los equipos de orientación educativa de la provincia de Huelva y su intervención en niveles no universitarios.

Colás y Buendía (1994) afirman que la investigación educativa parte de una situación problemática a la que el investigador no sabe dar respuesta con los conocimientos previos que posee.

Por tanto, es importante comprobar si esta institución educativa en Andalucía puede enfrentarse a la dura labor que le plantea el nuevo modelo de orientación que se refleja en la ley de educación andaluza. Por tanto, las cuestiones que constituyen el eje de nuestra investigación podían quedar formuladas de la siguiente manera:

- Conocer los datos de identificación: sexo, edad, titulación, experiencia en el puesto, desplazamientos realizados.

- Describir cómo se llevan a la práctica las funciones encomendadas por la administración y que caracterizan el trabajo de los equipos.

- Analizar las competencias que los profesionales de los equipos consideran importantes para llevar a cabo su intervención.

- Averiguar los contenidos prioritarios que se desprenden de las funciones que realizan los diferentes profesionales de los equipos.

Para clarificar las fases de investigación seguidas en este trabajo, las describiremos a continuación:

$1^{\mathrm{a}}$. Identificación de las dimensiones objeto de estudio y de los objetivos de la investigación.

$2^{\text {a }}$. Estudio y revisión de otras investigaciones relacionadas con nuestra temática. 
$3^{\mathrm{a}}$. Revisión de la literatura y elaboración de la fundamentación conceptual.

$4^{\mathrm{a}}$. Decisión y caracterización de la muestra.

$5^{\text {a }}$. Determinación, construcción, estudio piloto del cuestionario y elaboración definitiva del instrumento.

6 . Aplicación a la población seleccionada.

8 . Tratamiento de los datos.

$9^{\text {a }}$. Análisis y valoración de los resultados.

$10^{\text {a }}$.Conclusiones, aplicaciones y limitaciones de la investigación.

Diseño y análisis estadístico

El proceso de tabulación y clasificación de los datos obtenidos es una etapa esencial de la investigación.

Nuestros datos fueron sometidos a un proceso de análisis descriptivo. Este análisis parte del cálculo de frecuencias correspondientes a cada una de las variables posibles en los diferentes ítems del cuestionario y del cruce de las mismas mediante tablas de contingencia.

\section{Resultados}

En este apartado se incluye la información que se desprende de cruzar las siguientes variables:

Género y titulación.

Género y competencias para el puesto.

Modelos teóricos y relación con la titulación.

Años de experiencia y funciones. 
Género y titulación

Previo al análisis de datos que se van a cruzar, se presenta una reseña relativa a la distribución de la muestra por género. La figura 2 presenta una mayor participación en la muestra del género femenino que coincide con la presencia de la mujer en los órganos de orientación en el ámbito educativo. En la Tabla 2 se muestran los datos relativos a la relación existente entre género y titulación:

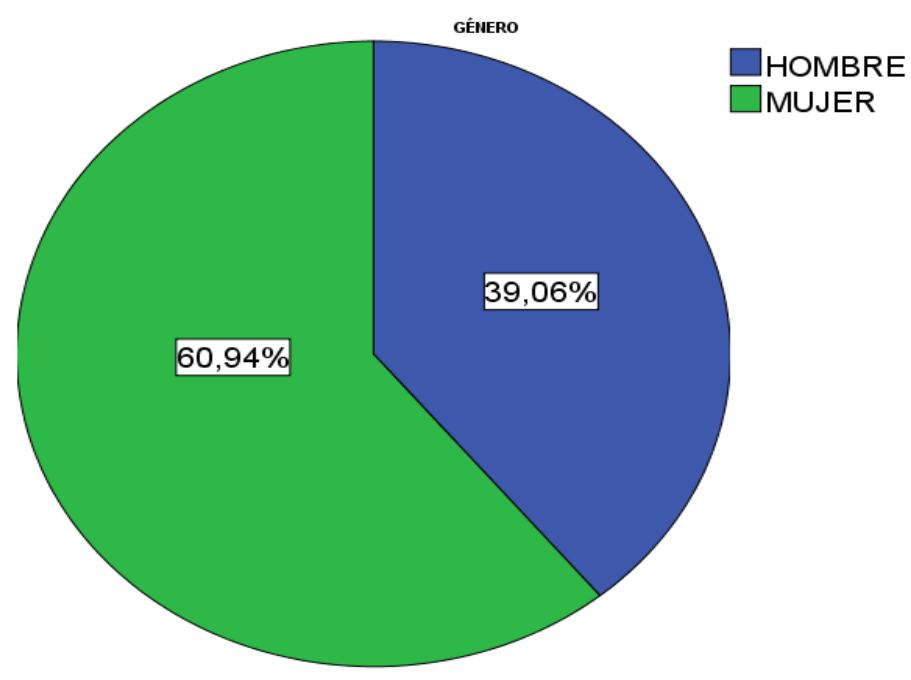

Figura 2. Género y Titulación

Tabla 2. Tabla de contingencia "Género-Titulación"

\begin{tabular}{|c|c|c|c|c|c|c|c|c|}
\hline & \multicolumn{7}{|c|}{ TITULACIÓN } \\
\hline & & Psicología & Pedagogía & Psicopedagogía & Magisterio & Medicina & Trabajo Social & Total \\
\hline \multirow{12}{*}{ 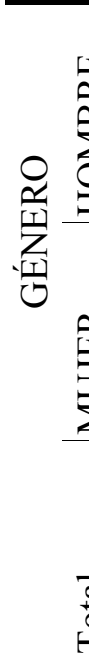 } & \multirow{4}{*}{$\begin{array}{l}\text { Recuento } \\
\% \text { Género } \\
\% \text { Titulación } \\
\% \text { del total }\end{array}$} & 6 & 5 & 3 & 3 & 7 & 1 & 25 \\
\hline & & $24,0 \%$ & $20,0 \%$ & $12,0 \%$ & $12,0 \%$ & $28,0 \%$ & $4,0 \%$ & $100,0 \%$ \\
\hline & & $26,1 \%$ & $26,3 \%$ & $37,5 \%$ & $75,0 \%$ & $77,8 \%$ & $100,0 \%$ & $39,1 \%$ \\
\hline & & $9,4 \%$ & $7,8 \%$ & $4,7 \%$ & $4,7 \%$ & $10,9 \%$ & $1,6 \%$ & $39,1 \%$ \\
\hline & \multirow{4}{*}{$\begin{array}{l}\text { Recuento } \\
\% \text { Género } \\
\text { \% \% Titulación } \\
\text { \% del total }\end{array}$} & 17 & 14 & 5 & 1 & 2 & 0 & 39 \\
\hline & & $43,6 \%$ & $35,9 \%$ & $12,8 \%$ & $2,6 \%$ & $5,1 \%$ &, $0 \%$ & $100,0 \%$ \\
\hline & & $73,9 \%$ & $73,7 \%$ & $62,5 \%$ & $25,0 \%$ & $22,2 \%$ &, $0 \%$ & $60,9 \%$ \\
\hline & & $26,6 \%$ & $21,9 \%$ & $7,8 \%$ & $1,6 \%$ & $3,1 \%$ &, $0 \%$ & $60,9 \%$ \\
\hline & \multirow{4}{*}{$\begin{array}{l}\text { Recuento } \\
\% \text { Género } \\
\text { \% Titulación } \\
\text { \% \% del total }\end{array}$} & 23 & 19 & 8 & 4 & 9 & 1 & 64 \\
\hline & & $35,9 \%$ & $29,7 \%$ & $12,5 \%$ & $6,2 \%$ & $14,1 \%$ & $1,6 \%$ & $100,0 \%$ \\
\hline & & $100,0 \%$ & $100,0 \%$ & $100,0 \%$ & $100,0 \%$ & $100,0 \%$ & $100,0 \%$ & $100,0 \%$ \\
\hline & & $35,9 \%$ & $29,7 \%$ & $12,5 \%$ & $6,2 \%$ & $14,1 \%$ & $1,6 \%$ & $100,0 \%$ \\
\hline
\end{tabular}




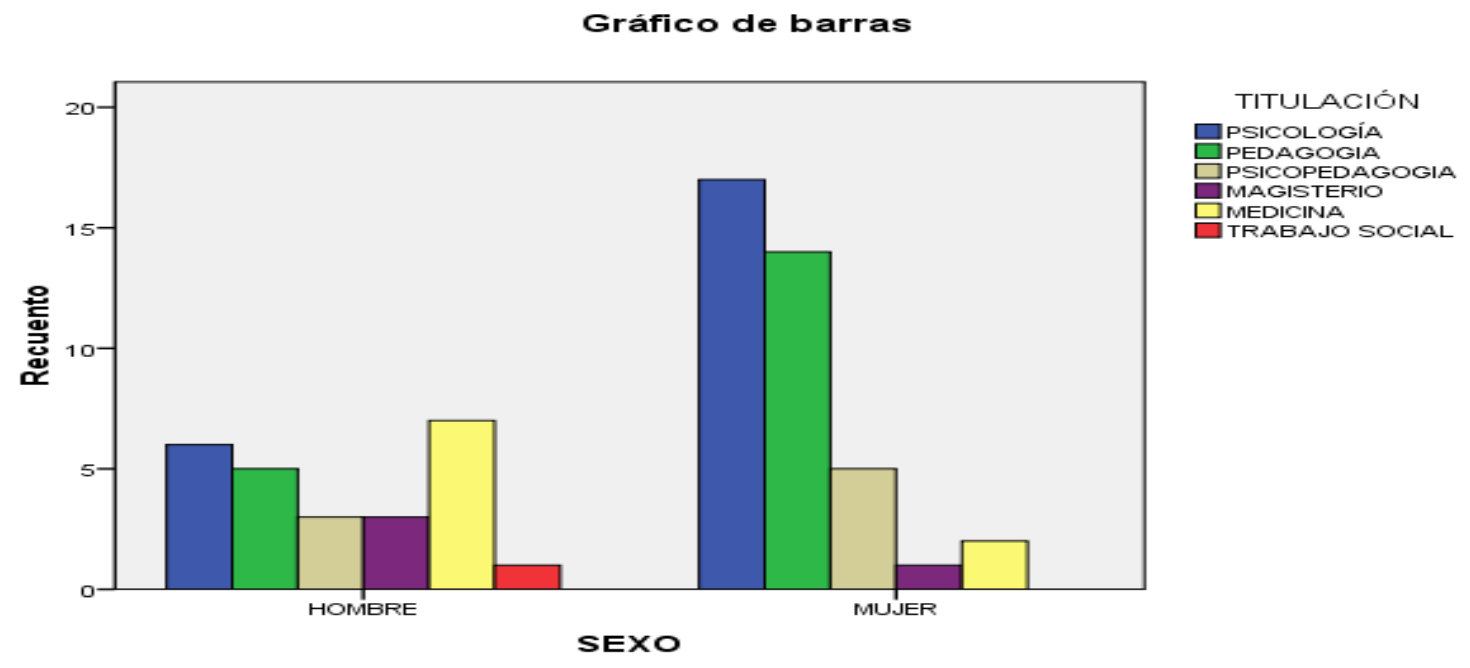

Figura 3. Relación de Género y Titulación

Destaca la presencia femenina en las titulaciones de psicología, pedagogía y psicopedagogía, que son los puestos que están ocupados por aquellos profesionales a considerar como orientadores/as. Con una presencia del $73,9 \%$ de la especialidad de psicología, un 73,7\% de pedagogía y 62,5\% de psicopedagogía, destacan ellas por encima del género masculino. Las demás ocupaciones (magisterio con un $75 \%$ y medicina con un $77,8 \%$ ) aparecen mayoritariamente ocupadas por hombres.

La presencia mayoritaria de las mujeres en los primeros niveles del sistema educativo español es el resultado de procesos históricos, políticos, económicos, religiosos y culturales. La feminización es un fenómeno que arranca a partir de la segunda guerra mundial y se concentra más en las carreras humanísticas, en sanidad y enseñanza.

\section{Género y competencias para el puesto}

En cuanto a las competencias (conocimientos teórico-prácticos, dinamizador y líder, habilidades sociales para trabajar en equipo, asesor y experto en la materia, capacidad de escucha y empatía, dominar las tic, dominio personal de situaciones problemáticas, otras) que son necesarias para el desempeño del puesto, existen discrepancias en el análisis por género. 
Tabla 3. Tabla de contingencia "Género-Competencias para el puesto"

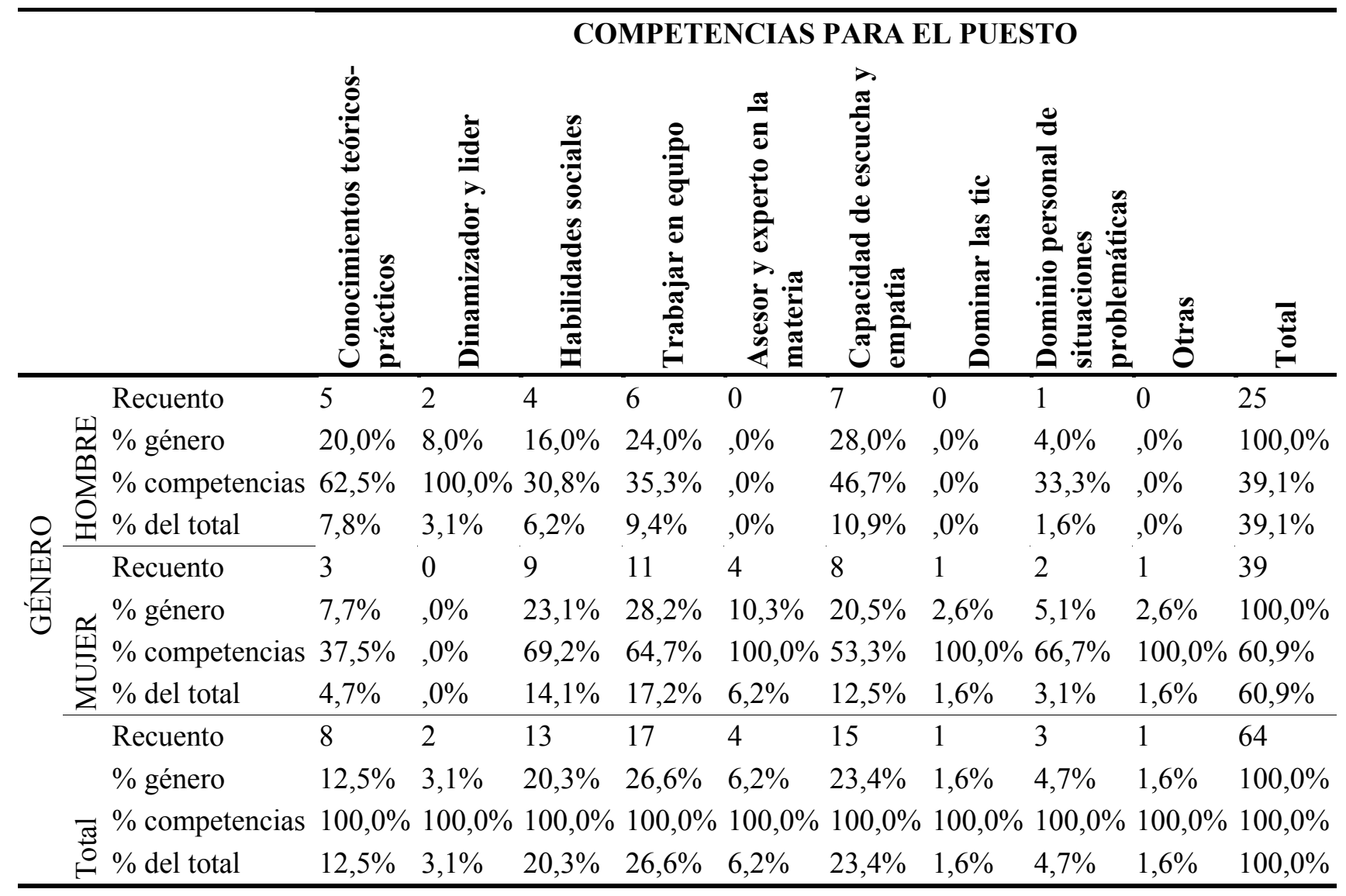

Ellas consideran que deben destacar entre las competencias del orientador/a la capacidad de trabajar en equipo, poseer habilidades sociales y de escucha y empatía, dejando en segundo término el ser asesor y experto en la materia o poseer conocimientos teórico-prácticos.

Mientras que ellos piensan que es primordial la capacidad de escucha y empatía, el trabajar en equipo y poseer conocimientos teórico-prácticos, indicando en segundo lugar la competencia en habilidades sociales y la capacidad de dinamización y liderazgo.

En ambos se detecta la importancia de ciertas actividades personales para desarrollar esta labor: la empatía, la capacidad de escucha y las habilidades sociales son bases necesarias para poder enfrentarse a la tarea orientadora donde las relaciones humanas afloran necesariamente. Asimismo el trabajo en equipo y la colaboración y coordinación entre su miembros es requisito imprescindible para ambos, aunque más en las mujeres. 


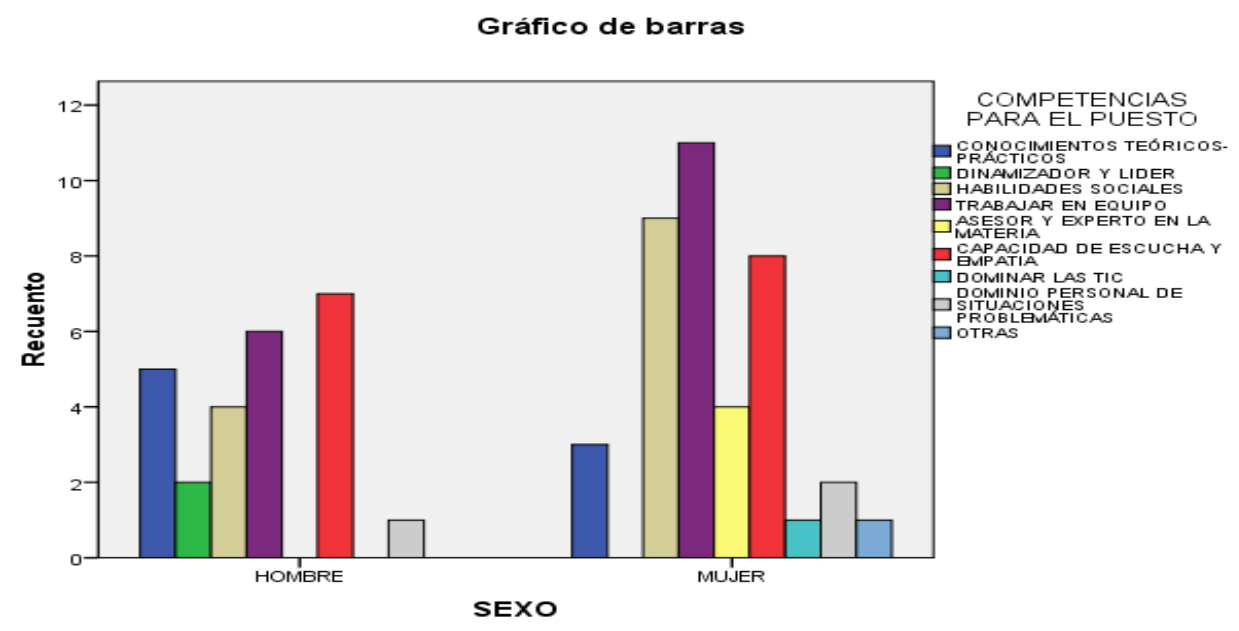

Figura 4. Titulación-Modelo Teórico

\section{Modelos teóricos y titulación}

Al cruzar la información relativa a los modelos teóricos y la relación con la titulación de los componentes de los EOEs, se obtienen los siguientes datos (Tabla 4).

Se han tomado como referencias de la distinción de los diferentes modelos las siguientes peculiaridades:

Modelo clínico: basado en el diagnóstico

Modelo psicopedagógico: actuación por programas, valorando la prevención

Modelo de consulta: servicio indirecto que se produce entre dos profesionales

Modelo ecléctico: combinación de los anteriores ajustándose al contexto y necesidades 
Tabla 4. Tabla de contingencia "Titulación-Modelo Teórico"

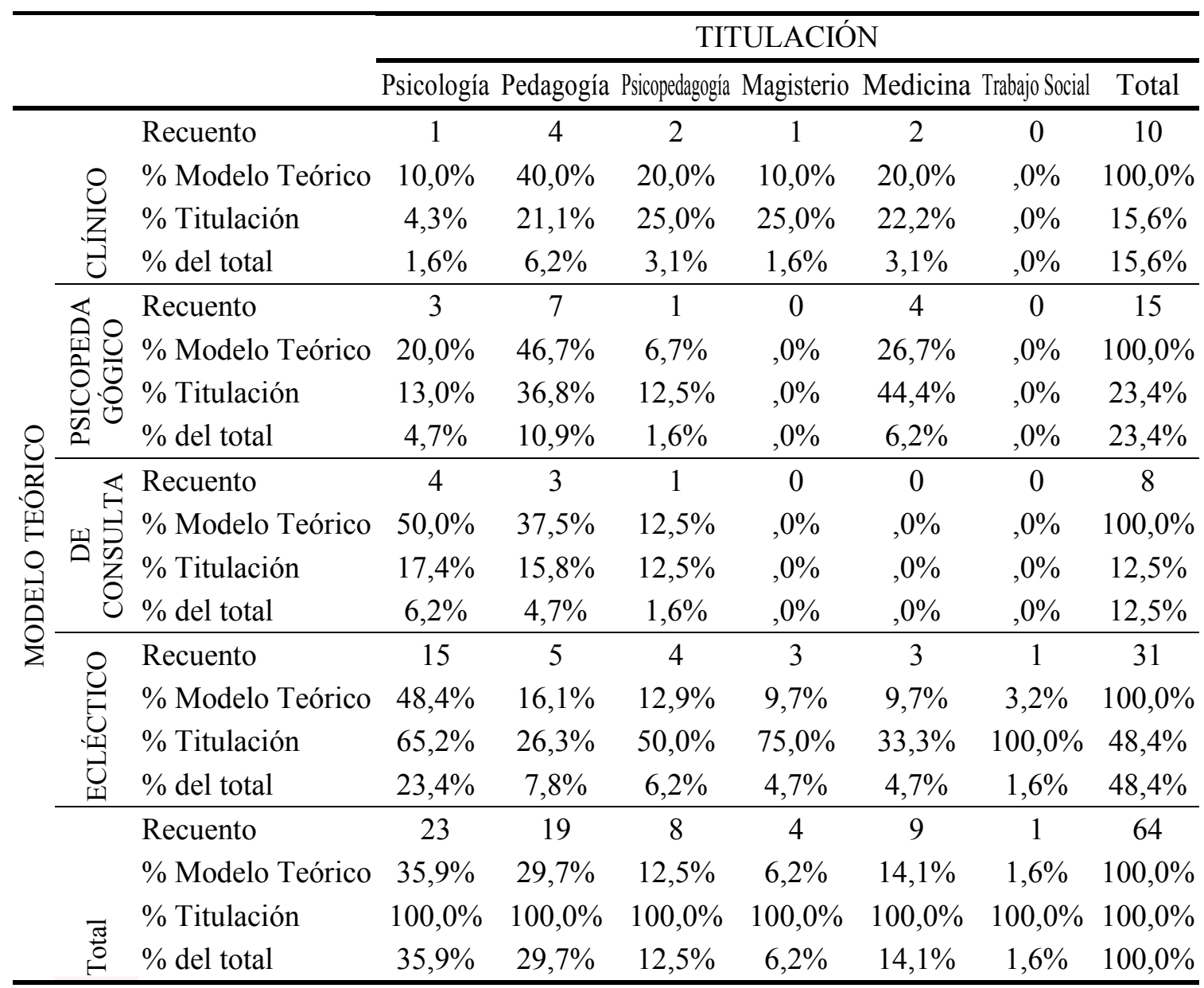

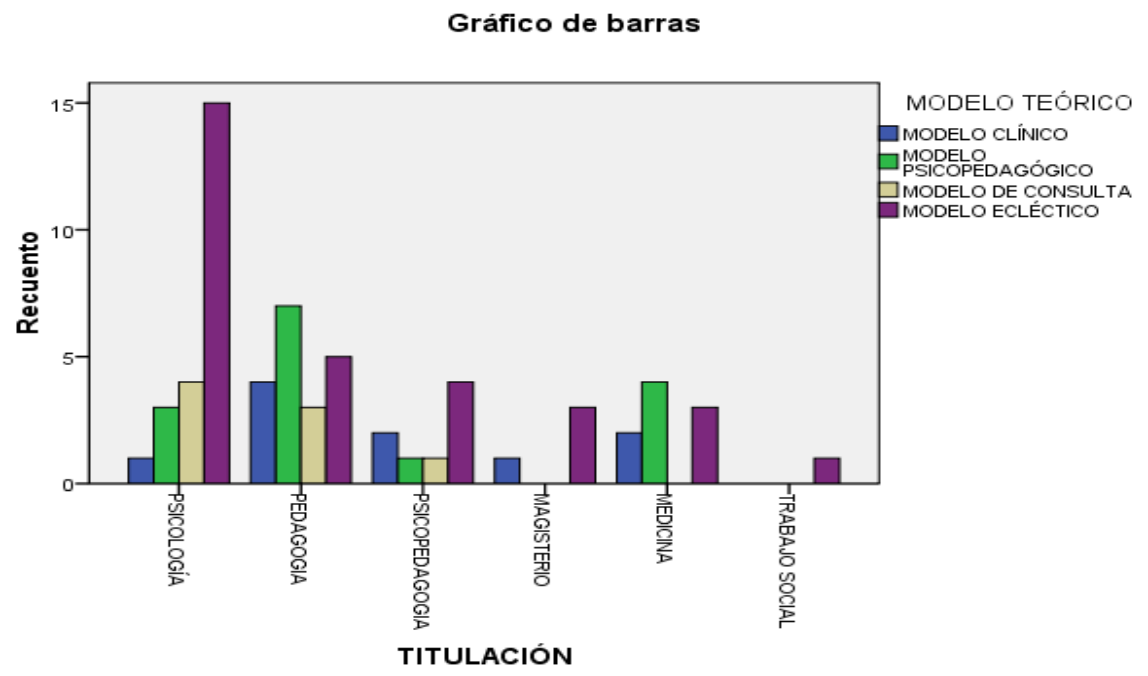

Figura 5. Titulación-Modelo Teórico 
Destaca el modelo ecléctico como posicionamiento habitual en la intervención de los distintos titulados que forman parte de los equipos de orientación educativa; no obstante, en un análisis más profundo, se observa que los titulados en pedagogía son más partidarios del modelo psicopedagógico, aunque entre estos titulados sorprende el dato relativo al modelo clínico.

En segundo término, y entre aquellos que tienen más directamente la función evaluativa, destaca el modelo psicopedagógico, posicionamiento que permite la evaluación psicopedagógica del alumnado, la intervención por programas con implicación del contexto y la prevención como intervención.

El modelo ecléctico es el más utilizado debido a la gran diversidad de funciones que deben de asumir los componentes de los equipos y adapta a cada actividad el modelo que le es más conveniente. Se ve paulatinamente cómo se va derivando hacia un modelo más psicopedagógico, centrado en la escuela y vivido desde dentro con el tutor y la comunidad educativa.

Años de experiencia y funciones

Por último, se presentan los datos sobre las funciones del EOE y los años de experiencia, dato este que también facilita información sobre el estado de la muestra.

Como se puede observar, se han establecido cuatro funciones del personal de los equipos de orientación educativa: as esora-miento, coordinación, intervención directa, evaluación. Por otra parte se han clasificado por tramos de 5 años de experiencia. Se puede establecer que la función de asesoramiento es la más usada entre los profesionales de la orientación seguida de la evaluación. Difiere de esta generalización el tramo de experiencia 6-10 años, quienes se posicionan fundamentalmente en el campo de la evaluación. 
Tabla 5. Tabla de contingencia "Años de Experiencia-Funciones EOE"

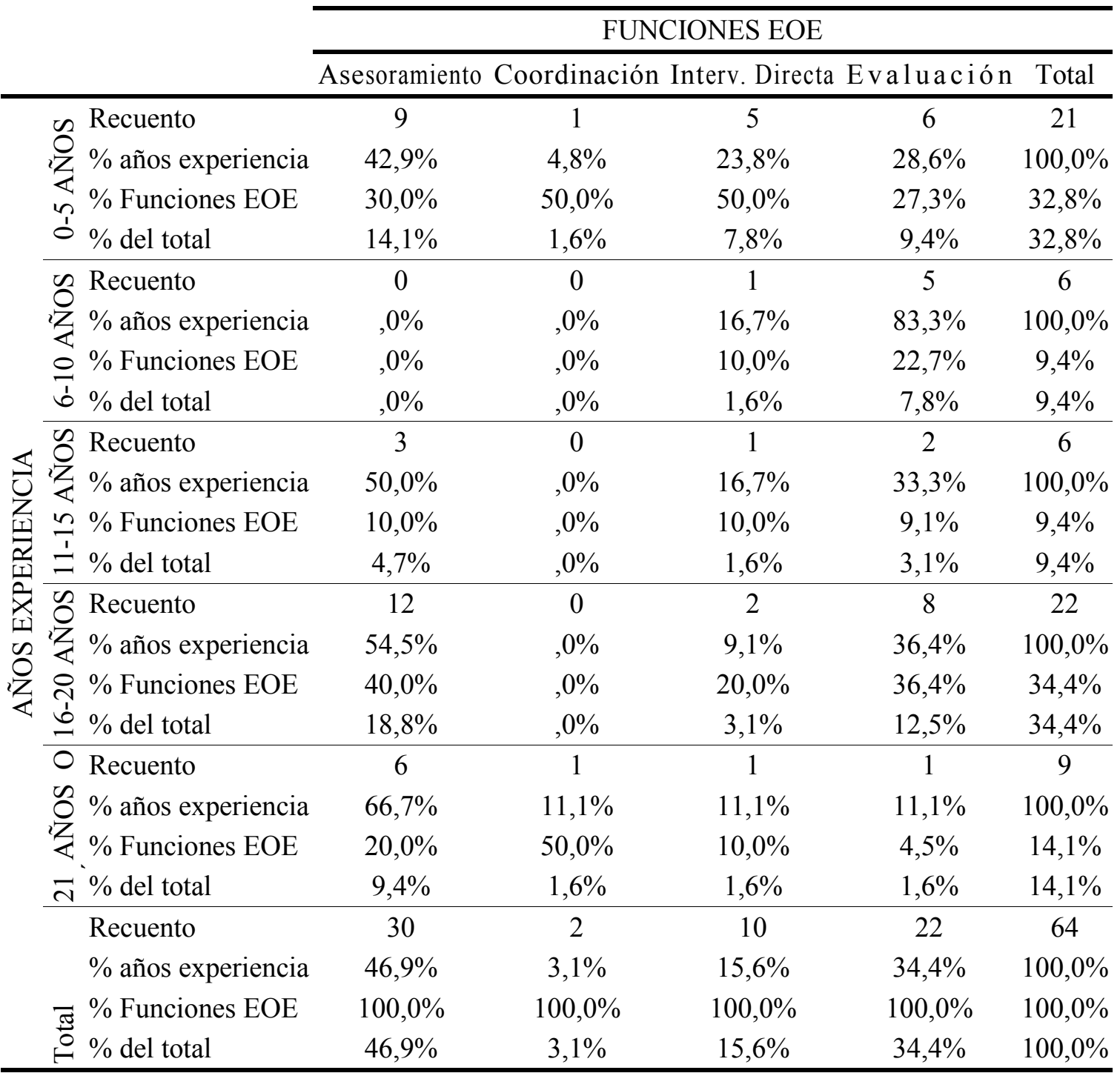

\section{Discusión y conclusiones}

Aunque en el apartado anterior ya han sido presentadas algunas de ellas, en éste, pretendemos recoger de manera más precisa las repercusiones y recomendaciones generales que se desprenden de la investigación después de la discusión de los datos.

Los servicios de orientación se presentan como una pieza clave en la mejora del proceso educativo. Todo alumno, familia y profesor tiene derecho a una orientación de calidad independientemente del tipo de centro. Desde ellos se aboga por un buen sistema de orientación desde la primera infancia hasta la madurez y se reivindica que se invierta en prevención y se potencie la orientación a lo largo de toda la vida. Se tiene la sensación que a 
pesar que la legislación prioriza la orientación en la práctica existe un abandono de la misma.

Esto supondría dotar al sistema de profesionales en los centros (Departamentos de Orientación en Infantil y Primaria) pero continuar con los Equipos cuyas labores serían complementarias. Cada servicio tendría establecidas sus funciones que serian distintas, pero complementarias. Replantearse los niveles que propugna el Plan de Orientación en Andalucía: tutoría, Departamento de Orientación y Equipo de Orientación Educativa (EOE), como elementos teóricamente interconectados, pero desvinculados en la práctica de la orientación educativa. Por ello se hace necesario fomentar la coordinación Departamento de Orientación (DO) y Equipo de Orientación Educativa (EOE) para no exista una línea tan descontentadas entre ambos estamentos que configuran la base de la orientación en nuestro sistema educativo.

Sería deseable garantizar el futuro de estas estructuras de apoyo en educación y su función de asesoramiento profesional en el ámbito escolar, fortaleciendo, mejorando e institucionalizando de manera consistente estos servicios de apoyo externo a nivel del sistema educativo. Constituye una forma de invertir en la mejora de la calidad educativa, una apuesta decidida por nuestro sistema educativo.

Es preciso hacer realidad el derecho de los alumnos a la orientación en todos los tramos y en todas las modalidades de escolarización. Para los centros de Infantil y Primaria los EOEs han constituido y constituyen un gran apoyo a su labor educativa y han representado y representan un recurso valioso para acometer procesos de toma de decisiones en la mejora educativa. Tanto en el cuestionario como en el grupo de discusión se pone de manifiesto el apoyo recibido de los centros y la valoración de su labor.

La administración educativa debe debatir y consensuar el papel que estos servicios desempeñan en nuestro sistema educativo analizando las Memorias de los centros, para definir y perfilar los objetivos y ámbitos prioritarios que deben de acometer y mediante una discusión interna diseñar un modelo que permita dotar de mayor coherencia al sistema de asesoramiento.

Es conveniente crear un modelo en el que los profesionales de la orientación atiendan a todo el alumnado con o sin necesidades educativas, asesoren al profesorado y orienten a las familias. Ofrecer una ayuda óptima en cada situación comportaría dotar de una gran ope- 
ratividad a estas estructuras, así como de recursos humanos y materiales suficientes. Se hace necesario valorar en qué medida el número de componentes con que cuenta cada sistema de apoyo, sus recursos y planes de actuación se adecuan a las necesidades reales de las zonas que atienden. Esta dotación aportaría a los equipos una gratificación y satisfacción hacia el desempeño de la difícil labor que supone la intervención psicopedagógica. Para poder desempeñar adecuadamente la gran heterogeneidad de funciones que se les asignan sería necesario ampliar significativamente el número de orientadores en todas las etapas educativas.

La mejora de las condiciones humanas y materiales y un justo reparto de las funciones a desempeñar crearía en los equipos la sensación de una valoración por parte de la administración. Este hecho justificaría, un panorama bien lejano, cuando se le otorgó a los antiguos Servicios de Orientación Escolar y Vocacional (SOEVs), allá en el año 1977, el objetivo de mejorar la calidad de la enseñanza, apostando por ellos como recurso necesario para lograrlo. Por tanto, habría que atender a ciertas condiciones laborales y profesionales que vienen afectando a los miembros de los sistemas de apoyo, revisar los incentivos y el tipo de reconocimiento profesional y, en general, las condiciones del puesto de trabajo, lo que revertiría sin lugar a dudas en la calidad del servicio prestado.

El aumento de su plantilla les permitiría actuar como asesores multidisciplinares y centro de recursos en todos los ámbitos y etapas no universitarias del sistema educativo. Se hace necesario mejorar y potenciar la coordinación interna de los sistemas de apoyo externo, posibilitando más estancia en la sede y permitiendo que se trabaje desde una perspectiva de equipo y con carácter interdisciplinar, flexibilizando los horarios para estar más acorde con la asistencia a los centros y la coordinación con la zona.

La necesidad de avanzar en la coordinación interservicios: servicios sociales, de salud, Centro Base de Atención al Minusválido, Departamentos de Orientación, inspección educativa, Centro de Profesorado (CEP), universidad; ha sido puesta de manifiesto de manera explícita a lo largo de toda la investigación, aunque también hay que señalar que se están dado pasos importantes en este terreno, pero insuficientes. La administración es la que debe potenciar estos encuentros y abandonar la voluntariedad de cada profesional a la hora de realizarlos. Una actitud abierta hacia el trabajo colaborativo favorecería un mayor acercamiento entre profesionales y evitaría la redundancia de actuaciones paralelas. 
Estos servicios con su intervención en el ámbito de la orientación profesional, como indica Repetto, Pena y Mudarra (2007: 173) "superarian el desafio de convertirse en un autentico recurso social para afrontar las múltiples elecciones y decisiones ocupacionales que cada individuo habrá de adoptar a lo largo de su vida".

Se requiere potenciar la formación de estos profesionales, intensificándola y diversificándola de acuerdo a las necesidades de cada zona y equipo; tanto aquella formación que favorece el desarrollo profesional de cada sujeto, como la que se centra en la capacitación y funcionamiento de los equipos de trabajo a nivel local, y del propio sistema de apoyo considerado en conjunto. La formación por áreas demandadas por ellos. La investigación y los grupos de trabajo deben de formar parte de su trabajo cotidiano y no plantearse la formación como actuaciones puntuales en momentos determinados. La formación en nuevas tecnologías y recursos informáticos iría encaminada a su perfil y deberían de apoyarse las iniciativas dirigidas a la investigación, renovación e innovación en el campo psicopedagógico y social, especialmente aquéllas que puedan derivarse de la colaboración con las universidades propuestas para el desarrollo profesional del orientador.

Teniendo presente las dificultades inherentes que conlleva el desempeño del rol de asesoramiento, la formación inicial y permanente de estos profesionales y servicios educativos debería contemplarse como un tipo de acción de primordial importancia en el funcionamiento y política de estas estructuras.

"La situación de los orientadores escolares en el sistema educativo, a caballo entre el trabajo directo de los alumnos y el trabajo de asesoramiento a los profesores y familia les permite estar en una situación privilegiada para ejercer la función de asesor, consultor y formador a la vez desde dentro y desde fuera" (Aguaded, Boza, Fondón, Ipland y Salas, 1997, p. 170) 


\section{Referencias}

Aguaded, M. C. (2009). Los equipos de orientación educativa en la provincia de Huelva. Análisis de la situación actual y prospectiva. Tesis Doctoral inédita.

Aguaded, M. C., Boza, A., Fondón, M. J., Ipland, J. y Salas, M. (1997). Ser profesor, ser tutor. Huelva: Hergué.

Ávila, J. A. (2009). El Plan de Orientación y Ación Tutorial en Educación Infantil y Primaria. Documento inédito.

Barrera, Á.; Durán, R.; González, J. y Reina, C.L. (2008). Guía para la elaboración del plan de orientación y acción tutorial en Educación Infantil y Educación Primaria. Sevilla. Junta de Andalucía, Consejería de Educación, Dirección General de Participación y Equidad en Educación.

Colás, M. P y Buendía, L (1992). Investigación educativa. Sevilla: Alfar.

Fernández Gálvez, J. (2003). Los Equipos y Departamentos de Orientación: Clave para el desarrollo de sus profesionales (buscando la magia del mago sin magia). Granada: Universidad de Granada (tesis doctoral).

Hernández Rivero, V. (2002). Sistema de apoyo externo a los centros educativos. La Laguna: Universidad de la Laguna (tesis doctoral).

Hernández Pina, F. (1988). Orientación escolar y equipos de apoyo psicopedagógico. En AA.VV. Jornadas sobre la Reforma del sistema educativo (pp. 173-187). Murcia: ICE de la Universidad de Murcia

JUNTA DE ANDALUCÍA (2007). Ley 17/2007, de 10 de diciembre, de Educación de Andalucía. Sevilla: BOJA, 252, 5-36.

Kerlinger, F. N. (1979). Behavioral research: a conceptual approach. Nueva Cork: Holt

López García, J. (1990). Estudio descriptivo-legal de los servicios institucionales de orientación educativa en España. Madrid: Editorial de la Universidad Complutense (tesis doctoral).

M.E.C. (2006). Ley Orgánica 2/2006, de 3 de mayo, de Educación. Madrid: BOE núm. 106; 17158- 17207.

Molina, D. y De Luca, C. (2009). Orientación integral en los centros educativos y el aula. En Electronic Journal of Research in educational Psychology, 19, 7(3), 1449-1460.

Pérez Serrano, M. C. (1988). La formación práctica del maestro. Madrid: Escuela Española.

Repetto, E., Pena, M. y Mudarra, M. J. (2007). Orientación de las competencias socioemocionales de los alumnos de educación secundaria. En Electronic Journal of 
Research in educational Psychology, 11, 5(1), 159-178.

Rodríguez Espinar, S. (1988). La orientación educativa y la calidad de la educación”. Bordón, $40(2), 235-255$.

Rodríguez Moreno, Ma . L. (1996). Orientación educativa. Barcelona: CEAC.

Rus, A. (1995). Institucionalización de la orientación educativa en Andalucía. Los servicios de apoyo a la escuela. Granada: Universidad de Granada (Tesis doctoral).

Rus, A. (1996). Tutoría, Departamento de orientación y Equipos de Apoyo. Granada: Servicios de Publicaciones de la Universidad de Granada.

Torres, M. G. (2000). Calidad del impacto y de la intervención de los grupos de orientación educativa. Estudio analítico descriptivo de sus actuaciones y estilos en la Comunidad Autónoma Andaluza. Granada: Universidad de Granada (Tesis Doctoral) 
María Cinta Aguaded et al.

[Página en blanco por razones de paginación] 